\title{
Acute Delirium and Sub-acute Cognitive Decline in a Patient with Probable Cerebral Amyloid Angiopathy Related Inflammation
}

\section{Yu-Ming Chen and Kuan-Fei Chen*}

Department of Neurology, China Medical University Hospital, China Medical University, Taiwan, Republic of China

\begin{abstract}
Cerebral amyloid angiopathy (CAA) is a well-known pathology of Alzheimer's disease among elderly individuals. CAA-related inflammation (CAAri) has been increasingly viewed as a rare presentation of CAA. We report the case a 65-year-old man who presented with subacute dementia and reversible delirium secondary to probable CAAri. His laboratory findings were negative except for elevated cerebrospinal fluid protein levels. Brain magnetic resonance imaging (MRI) revealed disseminated microbleeds in both the cerebral hemispheres on T2 weighted gradient images and prominent confluent T2-hyperintense white matter lesions in the bilateral temporo-occipital subcortical areas. Suspecting CAAri, we prescribed steroid pulse therapy with oral steroid tapering. The acute delirium and agitation improved after medication. However, the CAAri-induced damage to the brain function persisted, as evidenced in the follow-up clinical performance and serial Mini-Mental State Examination (MMSE).

The identification and accurate diagnosis of CAAri are important because the disease may respond to immunosuppressive therapy. In clinical settings, the detection of CAA with diffuse microbleeds in the brain is essential for making a tentative diagnosis of CAAri. Immunosuppressive therapy is the cornerstone of treatment. However, the optimal treatment duration is currently uncertain and warrants further investigation. In conclusion, the possible diagnosis of CAAri should always be considered when a patient presents with sub-acute cognitive decline and compatible MRI findings (T2-hyperintense lesions with diffuse microbleeds).
\end{abstract}

Keywords: Cerebral amyloid angiopathy-related inflammation; Cognitive decline

\begin{abstract}
Abbreviations: CAA: Cerebral Amyloid Angiopathy; CAAri: Cerebral Amyloid Angiopathy-Related inflammation; MRI: Magnetic Resonance Imaging; MMSE: Mini-Mental State Examination; ADLs: Activities of Daily Living; RPR: Rapid Plasma Regains; CSF: Cerebrospinal Fluid; APOE: Apolipoprotein; WMH: White Matter Hyperintensity
\end{abstract}

\section{Introduction}

Among elderly individuals, cerebral amyloid angiopathy (CAA) is a well-known pathology of Alzheimer's disease. CAA is characterized by amyloid $\beta$-peptide deposits within small to medium-sized blood vessels of the brain and leptomeninges [1]. CAA is usually asymptomatic, but it is an important cause of primary lobar intracerebral hemorrhage in elderly individuals. CAA may also present as transient ischemic attacks, incidental microbleeds through brain magnetic resonance imaging (MRI), focal seizures, visual disturbances, cognitive impairment caused by inflammatory leukoencephalopathy, and dementia [1]. CAA-related inflammation (CAAri) is being increasingly recognized as a rare complication of CAA. According to Castro Caldas's systemic review with 58 publications on 145 patients, the mean age of the first clinical manifestation was 66.9 years with slight male predominance $(53.5 \%)$. The most common clinical presentation of CAAri was cognitive dysfunction [2]. The clinical syndrome of CAAri is distinguished by subacute neurobehavioral symptoms, headaches and seizures [3]. We report the case of a man who presented with subacute dementia and reversible delirium secondary to probable CAAri. The aim of study highlights the typical clinical presentation and follow-up of cognitive function in the patient with probable CAAri.

\section{Case Presentation}

A 65-year-old, right-handed, previously independent man presented with the subacute onset of daily functional decline for 3 months. He would easily lose his way, and he failed to perform daily tasks such as counting, using light switches, and driving a car. He exhibited altered consciousness, with confusion and agitation. He had no systemic disease such as hypertension and diabetes mellitus. He had slowly progressive memory impairment for 2 years previously, but he remained independent in his activities of daily living (ADLs). Neurologic examination revealed prominent executive function impairment, poor short-term memory, progressive expressive aphasia, poor attention and behavioral changes (repetitive and meaningless behavior). No clear pyramidal signs were observed. The sub-acute cognitive decline greatly impaired his ADLs. Routine blood tests and surveys for the common causes of reversible dementia, such as vitamin B12, folic acid, and rapid plasma regain (RPR) revealed normal findings. Coagulation profiles and vasculitic and autoimmune screens were also normal.

A cerebrospinal fluid (CSF) study revealed clear fluid, and normal glucose levels, and normal white blood cell and red blood cell counts. The microprotein level was elevated $(130 \mathrm{mg} / \mathrm{dL})$. Tests and cultures for bacteria, viruses, and Cryptococcus were all negative. Electroencephalography disclosed diffuse slow waves over both hemispheres but no epilepticform discharge. Brain MRI revealed diffuse disseminated microbleeds in both cerebral hemispheres on T2-weighted gradient images. Abnormal patchy hyperintensity in the subcortical white matters and both periventricular regions were presented on T2-weighted images, without significant enhancement.

*Corresponding author: Kuan-Fei Chen,Department of Neurology, China Medical University Hospital, China Medical University, Taichung 40402, Taiwan, Tel:886975681634; E-mail: d11866@mail.cmuh.org.tw

Received May 08, 2017; Accepted May 27, 2017; Published May 30, 2017

Citation: Chen Y, Chen K (2017) Acute Delirium and Sub-acute Cognitive Decline in a Patient with Probable Cerebral Amyloid Angiopathy Related Inflammation. J Neurol Disord 5: 344. doi:10.4172/2329-6895.1000344

Copyright: () 2017 Chen Y, et al. This is an open-access article distributed under the terms of the Creative Commons Attribution License, which permits unrestricted use, distribution, and reproduction in any medium, provided the original author and source are credited. 
The bilateral occipital lobes and subcortical white matter were swollen with mild mass effect (Figure 1). The multifocal white matter hyperintensity lesions were asymmetric over bilateral parietal area. There was no superficial siderosis.

Apolipoprotein (APOE) $\varepsilon 4 / \varepsilon 4$ genetic testing was done and revealed positive finding afterwards.

Considering a tentative diagnosis of CAAri, we prescribed steroid pulse therapy for 5 days during the hospitalization. Subsequently, the patient's clinical condition was responsive to pulse therapy (Methylprednisolone $1000 \mathrm{mg} /$ day). The acute delirium, agitation and aggressive behavior were ameliorated during hospitalization period. We continued the immunosuppressive therapy with oral prednisolone (initial dose $30 \mathrm{mg} /$ day) for the further 3 months. Thereafter, we followed the patient by through clinical performance, and Mini-Mental State Examination (MMSE) as well as brain MRI scans.

Despite the clinical improvement with steroid pulse therapy in the acute stage, CAAri-induced damage to the brain persisted and caused permanent cognitive functional decline, as detected through serial MMSE scores (Table 1) and clinical performance over the course of 1 year. Follow-up brain MRI scans also revealed substantial resolution of the edema and inflammation after pulse therapy (Figure 1) compared with the acute-stage images. The patient still requires considerable assistance for most ADLs now.

\section{Discussion}

We report a typical case of subacute onset of cognitive decline secondary to CAAri. CAAri is a rare disease related to CAA, an
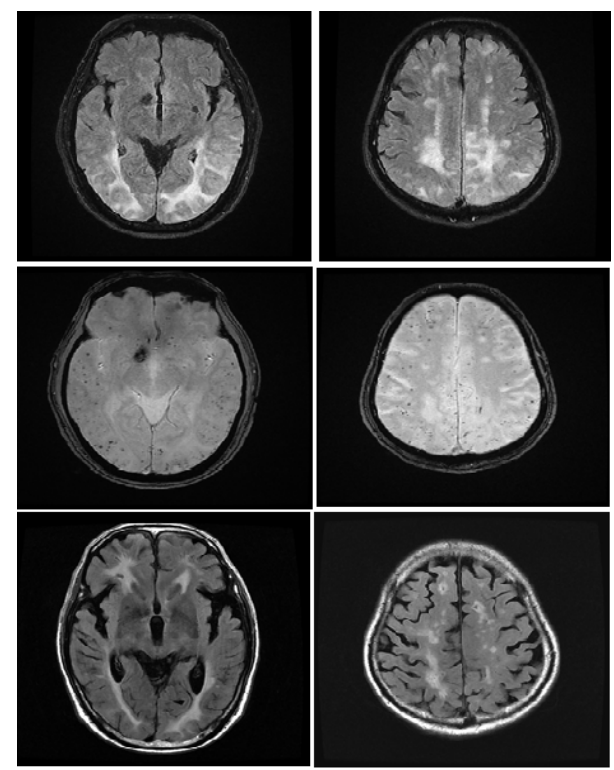

Figure 1: T2 FLAIR images showing confluent T2-hyperintense white matter lesions in the bilateral temporo-occipital areas. (3 and 4) Gradient-echo MRI showing multiple, obvious punctate hypointense cortical lesions caused by microbleeds. (5 and 6) Follow-up T2 FLAIR MRI at 3 months, which showed considerable resolution of $\mathrm{T} 2$ hyperintensity.

\begin{tabular}{|c|c|}
\hline Dates & Total MMSE Score (30) \\
\hline $2015 / 06 / 01$ & 11 \\
\hline $2015 / 07 / 15$ & 1 \\
\hline $2015 / 12 / 04$ & 4 \\
\hline $2016 / 10 / 03$ & 6 \\
\hline
\end{tabular}

Table: The patient's serial MMSE score. important risk factor for intracranial hemorrhage in elderly individuals. CAA is characterized by the deposition of $\beta$-amyloid protein in small to medium-sized blood vessels of the brain and leptomeninges, which may undergo necrosis or hemorrhagic conversion [1-5]. CAA can be related to genetic factors APOE $\varepsilon 2$ and $\varepsilon 4$ allele [3].

Chung and colleagues first suggested the following clinical criteria for the diagnosis of CAAri [4]: (1) acute or subacute onset of symptoms; (2) age $\geq 40$ years; (3) the presence of at least one of the following symptoms: headache, mental or behavioral change, focal neurological signs, or seizures; (4) MRI finding of patchy or confluent T2 signals; (5) evidence of CAA through gradient echo MRI or previous lobar hemorrhage; and (6) no neoplastic or infectious cause of this picture. In 2016, Auriel and colleagues further refined and operationalized these criteria to reflect previous experience with the clinical presentation, specific pattern of white matter hyperintensity $(\mathrm{WMH})$ distribution, and superficial siderosis as a type of bleeding manifestation of CAA [6]. Based on the Auriel's research, the brain MRI findings of our patient strongly supported the diagnosis of probable CAAri [6].

The essential step in the diagnosis of CAAri is the detection of CAA [7]. Microbleeds are typically located at the corticalsubcortical junction, more prominently in the posterior region [5]. Microbleeds present as a decreased or absent signal on T2 gradientecho sequences. However, microbleeds might not be observed in all CAAri cases according to Sakaguchi's review [8]. Brain biopsy should be considered if CAAri is highly suspected from clinical presentation despite absence of microbleeds on brain MRI [8]. Our patient's brain MRI findings revealed confluent areas of asymmetric T2 FLAIR white matter hyperintensities over the bilateral temporal and occipital lobes; and $\mathrm{T} 2$ gradient-echo diffuse microbleeds over the bilateral corticalsubcortical junction.

With respect to the APOE genotype, the presence of APOE ع4/ $\varepsilon 4$ genotype is a risk factor for CAAri [3]. Many authors found that inflammatory change in patients with CAA is more prevalent when the $\varepsilon 4 / \varepsilon 4$ genotype is present. According to series reviewed by Kinnecom et al. $\varepsilon 4 / \varepsilon 4$ genotype is found in $76.9 \%$ of patients with CAAri; in patients who have CAA without inflammatory change, the same genotype is occurring only $5 \%$ of the cases [9]. Further APOE $\varepsilon 4 / \varepsilon 4$ gene detection in our patient provided evidence of CAAri.

Considering a tentative diagnosis of CAAri, we prescribed steroid pulse therapy for 5 days during the hospitalization. Some studies have described a relationship between autoantibodies to the vascular amyloid $(\mathrm{A} \beta)$ and CAAri $[3,10]$. The reduced autoantibody levels correlate with clinical improvement. CSF analysis of our patient revealed normal findings except for elevated protein levels $(130 \mathrm{mg} / \mathrm{dL})$, suggesting an inflammatory cause. We couldn't test for CSF A $\beta$ in our facility.

The identification and proper diagnosis of CAAri are important because the disease may respond to immunosuppression. Piazza and colleagues discussed the immunological aspects of CAAri [10]. The treatment response is variable but generally good. Corticosteroids and immunosuppressants such as mycophenolate mofetil and methotrexate appear to be the drugs of choices in the literature [4]. The optimal duration of treatment is currently uncertain [4]. We treated our patient according to the clinical response, imaging findings and literature review experience. The relapse of CAAri has been reported, but the relapse generally responds well to the restarting of immunosuppressive drugs $[3,4]$.

Our case report has several limitations: (1) CSF A $\beta$ couldn't be checked in our facility, reduced CSF $A \beta$ with increased anti-A $\beta$ autoantibodies can be alternative evidence for the diagnosis of CAAri, 
Citation: Chen Y, Chen K (2017) Acute Delirium and Sub-acute Cognitive Decline in a Patient with Probable Cerebral Amyloid Angiopathy Related Inflammation. J Neurol Disord 5: 344. doi:10.4172/2329-6895.1000344

(2) CAAri is a rare disease with only one case in our clinical experience, which make the view one-sided, (3) the immunosuppression duration was empirical. In the literature, long-term efficacy of immunosuppression has not been evaluated. Future studies may try to search out the optimal strategy and duration of immunosuppression.

\section{Conclusion}

In summary, we presented a case of subacute onset of cognitive decline with delirium secondary to CAAri. The delirium status was resolved after immunosuppressive therapy. The notable points in our case were as follows: (1) the typical confluent T2 MRI hyperintense lesions over the bilateral temporal and occipital lobes with diffuse microbleeds; (2) the persistent impaired cognitive function sequelae, despite immunosuppressive therapy; and (3) APOE $\varepsilon 4 / \varepsilon 4$ gene detection in the blood (serum), which confirmed the diagnosis of CAAri. The early recognition of CAAri is crucial because immunosuppressive agents are potentially amenable. The possible diagnosis of CAAri should always be considered when a patient presents with subacute cognitive decline and compatible MRI findings (T2-hyperintense lesions with diffuse microbleeds).

\section{References}

1. Maia LF, Mackenzie IR, Feldman HH (2007) Clinical phenotypes of cerebral amyloid angiopathy. J Neurol Sci 257: 23-30.

2. Caldas CA, Silva C, Albuquerque L, Pimentel J, Silva V, et al. (2015) Cerebral amyloid angiopathy associated with inflammation: Report of 3 Cases and a systematic review. J Stroke Cerebrovasc Dis 24: 2039-2048.

3. Kirshner HS, Bradshaw M (2015) The inflammatory form of cerebral amyloid angiopathy or "Cerebral Amyloid Angiopathy-Related Inflammation" (CAARI) Curr Neurol Neurosci Rep 15: 54

4. Chung KK, Anderson NE, Hutchinson D, Synek B, Barber PA (2011) Cerebral amyloid angiopathy related inflammation: Three case reports and a review. $J$ Neurol Neurosurg Psychiatry 82: 20-26.

5. Rigney L, Sebire D, Cordato D (2015) Acute dysphasia and reversible cognitive decline in a patient with probable cerebral amyloid angiopathy-related inflammation. Case Rep Neurol Med 2015: 189581.

6. Auriel E, Charidimou A, Gurol ME, Ni J, Van Etten ES, et al. (2016) Validation of clinicoradiological criteria for the diagnosis of cerebral amyloid angiopathyRelated inflammation. JAMA Neurol 73: 197-202.

7. Savoiardo M, Erbetta A, Storchi G, Girotti F (2010) Case 159: Cerebral amyloid angiopathy-related inflammation. Radiology 256: 323-327.

8. Sakaguchi H, Ueda A, Kosaka T, Yamashita S, Kimura E, et al. (2011) Cerebral amyloid angiopathy-related inflammation presenting with steroidresponsive higher brain dysfunction: A case report and review of the literature. J Neuroinflammation 8: 116.

9. Kinnecom C, Lev MH, Wendell L, Smith EE, Rosand J, et al. (2007) Course of cerebral amyloid angiopathy-related inflammation. Neurology 68: 1411-1416.

10. Piazza F, Greenberg SM, Savoiardo M, Gardinetti M, Chiapparini L, et al (2013) Anti-amyloid $\beta$ autoantibodies in cerebral amyloid angiopathy-related inflammation: Implications for amyloid-modifying therapies. Ann Neurol 73: 449-458. 